Running head: The Fantasy Questionnaire

The Fantasy Questionnaire: A Measure to Assess Creative and Imaginative Fantasy David Weibel*, Corinna S. Martarelli, Diego Häberli, \& Fred W. Mast University of Bern, Switzerland

*Corresponding author:

David Weibel

Department of Psychology

University of Bern

Fabrikstrasse 8

CH-3012 Bern, Switzerland

Email: david.weibel@psy.unibe.ch

phone: +41316315434

fax: +41316318212 
Running head: The Fantasy Questionnaire

\begin{abstract}
This paper reports the construction and validation of a comprehensive self-report measure of fantasy. Unlike previous measures of fantasy, which focus on psychopathology, we conceive fantasy as a trait with positive connotation. Principal component analysis $(N=318)$ and confirmatory factor analyses $(N=345)$ were conducted using two sociodemographically diverse samples. The results provided support for a two-factor conceptualization of the construct, with the dimensions Imaginative Fantasy and Creative Fantasy. Imaginative Fantasy refers to vivid imagination and absorption in these images and daydreams. Creative Fantasy refers to the activity of using fantasy to create new ideas. The trait measure showed good internal consistency, test-retest reliability, discriminant and convergent construct validity, as well as incremental validity. Moreover, in three behavioral studies, we put fantasy scores in relationship with behavioral data in order to provide further proof of validity. A comprehensive measure of fantasy can contribute to our understanding of individual differences in inner experiences, creative processes, and problem solving.
\end{abstract}

Keywords: fantasy; imagination; imagery; creativity; assessment; questionnaire 


\section{The Fantasy Questionnaire: A Measure to Assess Creative and Imaginative Fantasy}

\section{Introduction}

"Without this playing with fantasy, no creative work has ever yet come to birth," wrote Carl Gustav Jung in 1921 (p. 82). Fantasy is a human function that strongly involves imagination (Lynn $\&$ Rhue, 1986) and thereby allows us to explore new worlds, think beyond existing paths, and discover new solutions. Moreover, fantasy allows us to go beyond perceptual reality and use cognitive abilities like counterfactual thinking, representation, and meta-cognition. Fantasy is essential from practical, artistic, technical, and scientific creativity (Mittelstrass, 2004). Fantasy is a pre-condition for any goal-oriented action, such as problem solving, art production, and identification of alternative solutions in complex fields (Barrett, 1992, 2010). Furthermore, fantasy concerns future interpersonal behavior and is a prerequisite to feeling empathy in human interactions (Barrett, 1992).

It is essential that we learn more about individual differences in fantasy across different domains of human life. However, to date, no questionnaire provides a comprehensive assessment of fantasy. The existing questionnaires concerning fantasy are linked to psychopathology and focus on imaginative aspects while ignoring creative or productive aspects of fantasy. Therefore, the present studies aim to develop a more comprehensive self-report measure to assess individual differences in fantasy. If successful, such a measure will not only contribute to a better understanding of individual differences in inner experiences, but may also result in applications in domains like problem solving or creative processes. This paper reports the evaluation of validity as well as reliability of a newly developed Fantasy Questionnaire.

\section{Definition of Fantasy}

The concept of "fantasy" is as fascinating as it is elusive. Etymologically, the English term "fantasy" is derived from the Greek " $\varphi \alpha v \tau \alpha \sigma i \alpha "$ (phantasia), which means appearance, outlook, illusion, and its Latin translation "imago." The concept of "fantasy" approximates those of representation, imagery, and imagination. The term "fantasy" has various definitions. In lay terms, fantasy is the process of imagining inner pictures and scenarios and thereby conceiving new ideas. 
In 1904, Rudolf Eisler proposed a definition of fantasy that distinguished between scientific and artistic fantasy, whereby scientific fantasy refers to the ability to mentally recombine different items and artistic fantasy to the activity of vividly imagining objects and situations in a pictorial format. In line with the concept of artistic fantasy, Lev Vygotsky (1930/2004) defined fantasy as creative imagination. Definitions that are more recent describe fantasy as "any departure from [...] reality" (Choi, Huang, Jeffrey, \& Baek, 2013, p. 1981), implying intense imaginative involvement (Lynn \& Rhue, 1986), often in order to escape reality (Bacon, Walsch, \& Martin, 2013), or as the productive use of imagination (Barrett, 2010).

Psychological research has yet to develop a precise definition of fantasy as a personality trait. Wilson and Barber (1983) introduced the term "fantasy-prone personality", which can be described as having an overactive imagination. Fantasy-prone individuals tend to report paranormal activities, spend a lot of time engaged in fantastical thinking, have vivid memories of their childhood, fantastical images, and clear daydreams that influence their physical sensations and perceptions. Highly fantasy-prone individuals tend to lose touch with reality and are often absorbed in mental activities. Fantasy proneness is conceptually related to openness to fantasy (cf. Merckelbach, Horselenberg, \& Schmidt, 2002), which is a facet of the openness to experience trait. Openness to fantasy is characterized by receptivity to inner world of imagination. Costa and McCrae (1992) stated that "individuals who are open to fantasy have a vivid imagination and an active fantasy life. They ... believe that imagination contributes to a rich and creative life" (p. 17).

Since fantasy is associated with an escape from reality, it has a rather negative connotation: Fantasy proneness is positively correlated with schizotypy (Merckelbach, Rassin, \& Muris, 2000), dissociation (Merckelbach et al., 2002), and psychopathological disorders (Rauschberg \& Lynn, 1995). Recently, however, Klinger, Henning, and Janssen (2009) cast doubt on fantasy proneness as a clinically relevant syndrome on its own. Indeed, fantasy can have a positive impact. For example, Taylor and Carlson (1997) found a significant positive relationship between fantasy experience (e.g., interacting with an imaginary friend) and theory-of-mind ability. Barrett (1992) pointed out that fantasy has beneficial aspects: Those with a high level of fantasy are often particularly creative 
and innovative. Fantasy abilities draw on vivid images and daydreams and allow one to think beyond existing paths and discover new information (cf. Eisler, 1904; Mittelstrass, 2004; Wilson \& Barber, 1983). In line with these findings and assumptions, Lynn and Ruhe (1986) found that fantasizers (fantasy-prone individuals) are more creative than non-fantasizers are.

Taken together, recent definitions suggest that the fantasy construct includes an imaginative (e.g., Bacon et al., 2013) and a creative (e.g., Barrett, 2010) component. Creativity is the process of finding new combinations of elements that are associated with each other (cf. Martindale, 2007; Mednick, 1962). Imagination is the ability to form pictorial experiences or sensations in our mind without the use of information from our senses (O’Connor \& Aardema, 2005). Imagination influences cognitive functions such as memory, attention, and perception (cf. Mast, 2009). Imagination and fantasy are related, but not identical. Imagination concerns the ability to recreate vivid quasi-perceptual memories in one's mind, whereas fantasy is referring to a product of imagination, for example, a vivid daydream consisting of a sequence of mental images. (e.g., Choi et al., 2013). In contrast, creativity is a practical activity with an objective result (e.g. an invention) that is in some way new, different, and effective. Unlike creativity, fantasy is not bound to a specific and objective output. However, these two concepts are not independent of each other: Various studies propose that fantasy is an imaginative way of discovering creative solutions and can help one to anticipate the realization of a creative construction (Bowers, 1979; Klinger, 1990; Lynn \& Rhue, 1986; Vygotsky, 1930/2004). Thus, fantasy can be viewed as a precondition of creativity or, in other words, creativity could be a manifestation of fantasy.

In the present study, in line with Vygotsky (1930/2004), Barrett $(1992,2010)$, and Wilson and Barber (1983), we define fantasy as the creative use of imagination. Thus, someone with a high propensity to fantasy has a vivid imagination that one uses and combines to form rich and absorbing inner worlds. In addition, one is able to make productive use of imagination in contexts such as artistic expression or problem solving. In line with Wilson and Barber, we consider fantasy a trait rather than a state. 


\section{Present Research}

The Fantasy Questionnaire aims to assess individual differences in the propensity to fantasy. We provide evidence of reliability and validity. Six phases of research were carried out: 1) We selected a pool of suitable items and conducted item analyses as well as principal component analysis in order to reduce the item pool and to examine the dimensionality of fantasy. We established a twodimensional structure (Imaginative Fantasy and Creative Fantasy). 2) This structure was confirmed with an additional sample using a confirmatory factor analysis. 3) Using the data collected in Phases 1 and 2, we provided normative data for different age groups. 4) We assessed test-retest reliability; the results suggested that the Fantasy Questionnaire is highly reliable with respect to temporal stability. 5) Convergent as well as discriminant construct validity was concluded from correlational analyses. 6) In three studies, we assessed criterion validity. Groups of artists and role players reported having a higher propensity to fantasy than did non-artists and non-role players. In addition, we collected behavioral data showing that individuals who score higher on fantasy perform better on a creativity test. Moreover, we explored the relationship between individual fantasy and subjective sense of boredom and absorption in a situation where participants had to wait for an experiment to begin. We could show that high fantasy scores correspond with being less bored and more absorbed in inner worlds, thoughts, and daydreams. All studies reported below were approved by the University's internal review board, and participants provided informed consent.

\section{Phase 1: Item Selection and Dimensionality}

In the first phase, we developed an initial item pool for subsequent data analysis. As recommended by Krohn and Hock (2007) and in line with other researchers' procedures (e.g., Denson, Pedersen, \& Miller, 2006), preexisting personality measures related to fantasy were used to create an initial pool of items. Defining "fantasy" as the creative use of imagination (see above), we expected fantasy to have an imaginative component (e.g., vivid mental images) and a creative component (e.g., the use of imagination to solve problems). We considered fantasy as a rather broad construct that includes facets like absorption, daydreaming, and fantasy proneness. To select 
appropriate items, we reviewed previous studies on the measurement of concepts related to these two components using the following keywords: fantasy, fantasy proneness, dissociation, intuition, daydreaming, mind wandering, absorption, inner experience, imagery, imagination, problem solving, divergent thinking, and creativity. In an ad hoc expert panel of advanced researchers and graduate students from the department, the selected items were discussed. To identify suitable items and to explore the dimensionality of fantasy, the resulting item pool (see Materials section below) was submitted to item analysis and principal component analysis.

\section{Method}

Participants. Three hundred and eighteen participants (51\% female, $49 \%$ male) completed the entire set of questions. The participants' average age was 32.5 years $(S D=12.27)$. University students recruited the participants as part of a psychology seminar. All participants of this particular course were advised to find 15 persons in their personal environment to fill out the questionnaire. To do so, we handed them a link to the questionnaire, which was accessible online. The dropout rate was 29\%. The participants' occupations were classified as: student (31\%), commercial profession (17\%), education (13\%), graduate occupation (13\%), technicians (11\%), social work (9\%), other (e.g., retired) (6\%). As in all other phases of research, we informed the participants that their responses would be anonymized.

Materials. Ninety items from 11 different scales were deemed useful for assessing fantasy as defined above and thus included in the initial item pool. As the existing items did not exhaustively cover the concept of fantasy, we generated 41 supplementary items. The supplementary items were mainly related to the creative component of fantasy (sample item: "Products of my fantasy, such as texts and drawings, come about almost automatically").

Procedure. Participants rated all items on five-point Likert scales ( 1 = strongly disagree, 5 = strongly agree), which were part of a multiple-page questionnaire that was accessible online. This questionnaire contained the 131 items described above and demographic questions (e.g. gender, age, occupation). 


\section{Results}

Item analyses. First, we evaluated the symmetry of the distribution of items: Thirty-two of the 131 items were either strongly negatively (skewness <-0.50) or strongly positively skewed (skewness > 0.50), indicating that distribution was not normal (cf. Lienert \& Raatz, 1998). As recommended in the literature on questionnaire construction (e.g., Clark \& Watson, 1995; Lienert \& Raatz, 1998; Moosbrugger \& Kelava, 2012), those 32 items were excluded from subsequent data analysis. Next, we calculated difficulty indices for the remaining items. The analysis revealed that the difficulty index of four items was below .20, indicating that participants consistently gave low ratings on these items. Following the recommendation by Bühl and Zöfel (2005), we deleted these items from the item pool. There were no difficulty indices above .80 .

Principal component analysis. In order to reduce the item pool and to explore the dimensionality of the construct, we conducted a principal component analysis (PCA) using the remaining 95 items. The PCA was followed by an oblique rotation, in order to allow correlations between the components. The measure of sampling adequacy (MSA) was 0.84, which is to be interpreted as "meritorious" (Kaiser \& Rice, 1974). Twenty-six components had an eigenvalue exceeding 1. A parallel analysis (cf. Horn, 1965) suggested the extraction of ten components. The eigenvalues of the first ten components after rotation were 15.14, 5.83, 3.09, 2.88, 2.48, 2.36, 2.06, $2.03,1.91$, and 1.85 . In contrast to the parallel analysis and in line with the eigenvalues, the scree test clearly suggested a two-component solution. Consequently, we computed and evaluated solutions for two to ten components. The two-components solution, which explained $45 \%$ of the total variance, was the most interpretable. It was difficult to interpret solutions with three and more factors due to the high secondary loadings. Moreover, some of the factors consisted of only two or three items with loadings higher than .30 and no items with loadings higher than .40 . Therefore, we chose the conceptualization with two dimensions. The first component explained $31.1 \%$ of the variance and contained 16 items with a factor loading higher than $.50($ Cronbach's alpha $=.89)$. The second factor explained additional $13.6 \%$ of the variance and contained 11 items with a component loading higher than .50 (Cronbach's alpha $=.90$ ). The two components explained $44.7 \%$ of the 
variance. In contrast to solutions with more than two components, in the two-component solution, no items had high loadings on both components.

\section{Discussion}

The first phase represented the starting point of the new fantasy measure. The aim was to gather suitable items and to examine the dimensionality of the construct. The results revealed two components, which accounted for almost half of the variance in the observed scores. An item was considered to load on a particular factor if the loading was above .50. The first component contained 16 items, which were related to the activity of vividly imagining and the ease of becoming absorbed in images and daydreams. We labeled this dimension "Imaginative Fantasy." The second component contained 11 items, which were related to the activity of using fantasy to create new ideas and inventions, as previously discussed by Barrett $(1992,2010)$. We labeled this dimension "Creative Fantasy."

\section{Phase 2: Replication of the Factor Structure}

To replicate the two-component solution revealed by the principal component analysis in Phase 1, a confirmatory factor analysis was conducted using a separate sample that was recruited online.

\section{Method}

Participants. Three hundred and forty-five respondents (66\% female, $34 \%$ male) completed an online questionnaire with no particular incentive given. The respondents' average age was 36.43 years $(S D=17.11)$. Their occupations were classified as: student $(39 \%)$, graduate occupation $(16 \%)$, commercial profession (13\%), education (8\%), technicians $(7 \%)$, social work $(3 \%)$, other (e.g., retired) (14\%). The participants were recruited by means of advertisement in an internal university publication where the link to the questionnaire was published. Furthermore, information about the study and a corresponding link was provided on the institute's homepage as well as in social media: The authors, the members of the regarding institute, as well as the university posted the link on Facebook. 
Materials and analysis. Participants completed the 27 items that were chosen based on the results of Phase 1 . The participants rated the items on a five-point Likert scale $(1=$ strongly disagree, 5 = strongly agree). A confirmatory factor analysis was conducted on the 27 items. We specified the same two-factor model consisting of an Imaginative Fantasy and a Creative Fantasy factor.

\section{Results and Discussion}

In order to test whether the two-factor solution fit the data, we used structural equation modeling with AMOS (Arbuckle, 2012). Maximum likelihood estimates were used. The model fit indices were $\operatorname{good}\left(\chi^{2}=303.86 ; d f=281 ; p=.17 ; C F I=.99 ; R M S E A=.02\right)$. Furthermore, all path coefficients were significant. Thus, in Phase 2, we were able to replicate the two-factor structure identified in Phase 1. The final questionnaire is presented in table 1. 


\section{Table 1}

\section{Items of the Final Questionnaire}

\begin{tabular}{|c|c|c|}
\hline Fantasy Dimension & Item & Source \\
\hline \multirow[t]{16}{*}{ Imaginative Fantasy } & Many of my fantasies have a realistic intensity. & CEQ, Merckelbach et al. (1999) \\
\hline & Many of my fantasies are often just as lively as a good movie. & CEQ, Merckelbach et al. (1999) \\
\hline & I am never bored because I start fantasizing when things get boring. & CEQ, Merckelbach et al. (1999) \\
\hline & When I think of something cold, I actually get cold. & CEQ, Merckelbach et al. (1999) \\
\hline & $\begin{array}{l}\text { In my daydreams I can hear the sound of a tune almost as clearly as if I } \\
\text { were actually listening to it. }\end{array}$ & LSHS, Launay \& Slade (1981) \\
\hline & Sometimes my thoughts seem as real as actual events in my life. & LSHS, Launay \& Slade (1981) \\
\hline & The sounds I hear in my daydreams are usually clear and distinct. & LSHS, Launay \& Slade (1981) \\
\hline & $\begin{array}{l}\text { Sometimes I remind apprehensive or fearful long after watching a scary } \\
\text { movie. }\end{array}$ & ITQ, Witmer \& Singer (1998) \\
\hline & $\begin{array}{l}\text { Sometimes I become so involved in a daydream that I'm are not aware of } \\
\text { things happening around me. }\end{array}$ & ITQ, Witmer \& Singer (1998) \\
\hline & $\begin{array}{l}\text { I'm good at blocking out external distractors when I'm involved in } \\
\text { something. }\end{array}$ & ITQ, Witmer \& Singer (1998) \\
\hline & My daydreams are often stimulating and rewarding. & IES, Huba et al. (1981) \\
\hline & I am the kind of person whose thoughts often wander. & IES, Huba et al. (1981) \\
\hline & I don't like to waste my time daydreaming. (R) & NEO-PI, Costa \& McCrae (1992) \\
\hline & $\begin{array}{l}\text { I have gone through the motions of living while the real me was far away } \\
\text { from what was happening to me. }\end{array}$ & SDT, Steinberg \& Schnall (2000) \\
\hline & $\begin{array}{l}\text { If I wish I can imagine (or daydream) some things so vividly that they hold } \\
\text { my attention as a good movie or story does. }\end{array}$ & TAS, Tellegen \& Atkinson (1974) \\
\hline & $\begin{array}{l}\text { When I read a book, the feelings of the character with whom I identify } \\
\text { influence my own mood. }\end{array}$ & Self-generated \\
\hline \multirow[t]{11}{*}{ Creative Fantasy } & A really original idea sometimes develops from a really fantastic dream. & IES, Huba et al. (1981) \\
\hline & Sometimes I think about new inventions. & Self-generated \\
\hline & I am a creative person. & Self-generated \\
\hline & I have been told to have a lot of fantasy. & Self-generated \\
\hline & My ideas are usually considered as very creative. & Self-generated \\
\hline & $\begin{array}{l}\text { Products of my fantasy such as texts and drawings generate themselves } \\
\text { almost automatically. }\end{array}$ & Self-generated \\
\hline & $\begin{array}{l}\text { I solve tasks in different ways, i.e. in unexpected, surprising and } \\
\text { inconventional ways. }\end{array}$ & Self-generated \\
\hline & I take the time to express my fantasies. & Self-generated \\
\hline & I have many ideas that are unsual and novel. & Self-generated \\
\hline & I can "think around" obtacles and find new solutions. & Self-generated \\
\hline & I have a lot of fantasy. & Self-generated \\
\hline
\end{tabular}

Note: $\mathrm{R}=$ Reversed coded. 


\section{Phase 3: Normative Data}

To provide normative data, we pooled the data gathered in Phases 1 and 2.

\section{Method}

Participants. The sample consisted of 663 participants (62\% female, 38\% male). Their average age was $34.5(S D=15.08)$. Their occupations were classified as: students $(35 \%)$, commercial profession (15\%), graduate occupation (13\%), education (12\%), technicians (9\%), social work (6\%), other (e.g., retired) (10\%).

\section{Results and Discussion}

We calculated mean values for the items of each dimension, in order to provide a better comparability of the two dimensions. Overall, the mean value for Imaginative Fantasy was 3.14 $(\mathrm{SD}=0.70)$ and that for Creative Fantasy was $3.25(S D=0.73)$. The two dimensions were correlated, $r(663)=.51, p<.001$. Table 2 shows the means and standard deviations for the different age groups.

Table 2

Mean values for different age groups.

\begin{tabular}{lccc}
\hline Subscale & Age Group & M & SD \\
\hline Imaginative Fantasy & $<20(n=41)$ & 3.24 & 0.63 \\
& $21-30(n=293)$ & 3.17 & 0.69 \\
& $31-40(n=133)$ & 3.15 & 0.72 \\
$41-50(n=50)$ & 3.15 & 0.68 \\
$51-60(n=67)$ & 3.11 & 0.68 \\
$>60(n=57)$ & 3.00 & 0.70 \\
Treative Fantasy & $<20(n=41)$ & 3.34 & 0.84 \\
& $21-30(n=293)$ & 3.31 & 0.70 \\
& $31-40(n=133)$ & 3.19 & 0.79 \\
$41-50(n=50)$ & 3.17 & 0.69 \\
$51-60(n=67)$ & 3.29 & 0.68 \\
$>60(n=57)$ & 3.10 & 0.69 \\
& Total $(\mathrm{N}=663)$ & 3.25 & 0.73 \\
\hline Note: 22 participants did not report their age & &
\end{tabular}


We found weak negative correlations between age and Imaginative Fantasy, $r(641)=-.10, p$ $=.01$, and age and Creative Fantasy, $r(641)=-.08, p=.04$. We found a difference between women $(M=3.21, S D=0.70)$ and men $(M=3.04, S D=0.71), t(642)=2.88, p=.002, d=0.24$, with respect to Imaginative fantasy, but no such difference with respect to Creative Fantasy (women: $M$ $=3.25, S D=0.73$, men: $M=3.27, S D=0.73), t(642)=-0.29, p=.77, d=0.03$.

\section{Phase 4: Test-Retest Reliability}

In Phase 4, we aimed to provide evidence for the temporal stability of the measure. A cohort of students in an undergraduate psychology course completed the Fantasy Questionnaire twice, with a 12-week interval between administrations.

\section{Method}

Participants. The sample consisted of undergraduate students, with 240 participants at Time 1 and 197 participants at time 2. We found 115 matching questionnaires (83\% from females, $17 \%$ from males, whose average age was 21.8 years, $S D=4.97)$. The participants completed a paper-and-pencil questionnaire as part of a course. Participants obtained an individual code, which allowed us to match the questionnaires from the two time points.

Material. We used the Fantasy Questionnaire from Phases 1 and 2, which consisted of 27 items. The participants rated the items on a five-point Likert scale $(1=$ strongly disagree, $5=$ strongly agree). We computed the respective means to obtain a score for each dimension. Both subscales demonstrated good internal consistency: Cronbach's alpha was .88 for the Imaginative Fantasy subscale and .90 for the Creative Fantasy subscale.

\section{Results and Discussion}

The 12-week test-retest reliability of both subscales was $\operatorname{good}(r=.79, p<.01$, for the Imaginative Fantasy subscale; $r=.82, p<.01$, for the Creative Fantasy subscale), which indicates that the Fantasy Questionnaire is stable over time. Moreover, $t$-tests for dependent samples yielded no differences in the means for Timepoint 1 or Timepoint 2 , not for Imaginative Fantasy $\left(M_{t l}=\right.$ $\left.3.05, S D_{t 1}=0.63 ; M_{\mathrm{t} 2}=3.05, S D_{t 2}=0.66 ; t(107)=0.05, p=.96, d<0.001\right)$, or Creative Fantasy $\left(M_{t 1}=3.24, S D_{t 1}=0.55 ; M_{\mathrm{t} 2}=3.23, S D_{t 2}=0.63 ; t(107)=0.25, p=.80, d=0.02\right)$. 


\section{Phase 5: Evidence of Construct Validity}

In the fifth phase, we analyzed the relationship between the two fantasy dimensions and theoretically relevant concepts namely, Big Five personality traits, openness to fantasy, empathy, imagery, tendency towards hallucinatory experiences, depersonalization, intuition, absorption, and creative self-efficacy.

\section{Method}

Participants. The participants were the same as those in Phase 4. They completed a number of questionnaires (described below) either at Timepoint 1 or Timepoint 2. In addition, two additional samples completed measures assessing openness to fantasy and creative self-efficacy (openness to fantasy: $N=122 ; 70 \%$ female, $30 \%$ male, average age 24.67 years, $S D=6.54$; creative self-efficacy: $N=133 ; 67 \%$ female, $33 \%$ male, average age 27.11 years, $S D=9.93$ ). In the two additional samples, most of the participants were students (74\% and 67\%, respectively).

\section{Materials}

Fantasy Questionnaire. We used the Fantasy Questionnaire from Phases 1 and 2. The mean response scores for the two dimensions were computed.

Big Five personality traits. We used Borkenau and Ostendorf's (1993) short form of the Big Five Personality Inventory-Revised (NEO-PI) (Costa \& McCrae, 1992) to assess the relation between fantasy and personality. The inventory measures personality in terms of the five dimensions extraversion (e.g., "I am spontaneous'), openness to experience (e.g., "'I often try new and foreign foods'), conscientiousness (e.g., 'I pay my debts promptly and in full’'), neuroticism (e.g., "Frightening thoughts sometimes come into my head"), agreeableness (e.g., "I believe that most people will take advantage of you if you let them'"). The scale consists of 60 items and was frequently used in the past. Its reliability, validity and dimensionality have been confirmed several times (cf. De Raad, 1998). The participants rated each item on a 5-point Likert scale (1 = strongly disagree, 5 = strongly agree). We computed a mean item response score for each participant for each dimension. Internal consistency of each dimension was sufficient. Cronbach's alpha were .79 
(extraversion), .85 (openness to experience), .87 (conscientiousness), .85 (neuroticism), and .74 (agreeableness).

Openness to fantasy. In addition to the short form of the openness to experience scale, we also administered the openness to fantasy scale, which is an openness scale in the full version of the NEO-PI (Costa \& McCrae, 1992). It consists of eight items (e.g., " I have a very active imagination') and was previously found to be reliable and valid (cf. De Raad, 1998; Costa \& McCrae, 1992). A mean item response score was computed for each participant. Cronbach's alpha was .78.

Empathy. We administered the Toronto Empathy Questionnaire (TEQ; Spreng, McKinnon, \& Levine, 2009) to investigate the relationship between fantasy and empathy. The TEQ is a short tool to assess empathy. It consists of 16 items (e.g., " I can tell when others are sad even when they do not say anything"). The items are rated on a five-point Likert scale $(1=$ never, $5=$ often $)$. Previously, the TEQ demonstrated strong convergent validity, good internal consistency and high test-retest reliability (Spreng et al.; Totan, Doğan, \& Sapmaz, 2012). We computed the mean value over all items. Cronbach's alpha was .80.

Mental Imagery. We assessed the participants' visual imagery skills with the Vividness of Mental Imagery Questionnaire (VVIQ; Marks, 1973). The VVIQ is the most frequently used measure to assess individual differences in vividness of mental images. The scale contains 16 items. It was shown to be reliable and was carefully validated (e.g. Richardson, 2013). For example, Rodway, Gillies, and Schepman (2006) and Gur and Hilgard (1975) found that participants scoring high on the VVIQ were better in detecting salient changes in pictures when compared to participants scoring low on the VVIQ; these results suggest that self-rated visual imagery as measured with the VVIQ can predict successful recall of visual information. Also, recent fMRI studies support the validity of the questionnaire. For example, Amedi, Malach and Pascual-Leone (2005) found a strong positive correlation between subjective vividness of visual imagery - as measured by the VVIQ - and deactivation in auditory area A1. It has been shown that the VVIQ is 
not particularly vulnerable to a response bias (e.g. McKelvie, 1995). Furthermore, previous studies (e.g. Campos, 2011; Campos, \& Pérez-Fabello, 2009) report that the VVIQ is correlated with other measures of imagination such as the Betts Questionnaire Upon Mental Imagery, the Object-Spatial Imagery Questionnaire, or the Gordon Test of Visual Imagery Control.

Participants were asked to create vivid mental images of four different scenes and then rate the amount of detail in the imagined scenes on a five-point Likert scale $(1=$ no picture at all, I only "know" that I think about an object, 2 = vague and gloomy, $3=$ reasonably clear and vivid, $4=$ clear and halfway vivid, $5=$ crystal clear and as vivid as normal view). A mean score was computed for each participant. Cronbach's alpha was .87 .

Sustaining fantasy. Sustaining fantasies are a means to ameliorate intense negative affect or to restore self-esteem (Zelin et al., 1983). We used the aesthetics subscale of the Sustaining Fantasy Questionnaire (SFQ) to investigate a possible relationship between fantasy and mood changes. According to Zelin et al., sustaining fantasies ameliorate intense negative affect or they help to restore self-esteem. Zelin et al. indicate high reliability and validity. However, they point out that most subscales of the questionnaire are clinical measures and therefore they may be less suitable for our purposes. Exclusively the aesthetics subscale can be used for healthy participants (Zelin et al.). Participants were asked to rate a number of sentences beginning with "It brightens my mood, when I . . " (e.g., "think about beautiful art work") on a five-point Likert scale (1 = strongly disagree, 5 = strongly agree $)$. The overall mean served as the SFQ score. Cronbach's alpha was .76.

Hallucination. We administered the Launay Slade Hallucination Scale (LSHS; Launay \& Slade, 1981) to assess a possible relationship between fantasy and hallucinatory experiences. Although the scale is frequently used in clinical settings, it is also an appropriate measure to assess predisposition to hallucinations in normal individuals (e.g.Ranking, \& O’Carroll, 1995). However, floor effects were observed with healthy participants when testing pathology-related items (e.g., "I have been troubled by hearing voices in my head") (cf. Launay \& Slade, 1981). Therefore, we only included the six items that were the least related to psychopathology and for which Waters, Badcock, and Maybery (2003) did not find such floor effects (e.g., "Sometimes I can see the face of 
a person in front of me, even though there is nobody there"). The participants rated the items on a five-point Likert scale $(1=$ strongly disagree, $5=$ strongly agree $)$. The overall mean served as the LSHS score. Cronbach's alpha was .77.

Depersonalization. We administered the Steinberg Depersonalization Test (SDT; Steinberg \& Schnall, 2000) to investigate a possible positive relationship between fantasy and depersonalization. The SDT assesses signs of dissociation, depersonalization, and loss of selfcontrol. It is widely used and was found to be valid and reliable (e.g. Mula et al., 2008). We included all 15 items of the SDT (e.g., "I have felt as if words flowed from my mouth, but they were not in my control"). The participants rated each item on a five-point Likert scale $(1=$ never, 5 = always). A mean score was computed. Cronbach's alpha was .88.

Intuition. Pretz and Totz (2007) analyzed the items of Pacini and Epstein's (1999) RationalExperiential Inventory and identified three factors, one of which was labelled intuition ability (IA). We administered the IA subscale to investigate the possible relationship between intuition and fantasy. The IA subscale consists of ten items (e.g., "If I were to rely on my gut feelings, I would often make mistakes") and Pretz et al. (2014) could demonstrate validity and internal consistency of the scale. The participants rated the items on a five-point Likert scale $(1=$ strongly disagree, $5=$ strongly agree). We computed a mean score. Cronbach's alpha was .86.

Absorption. We administered the Tellegen Absorption Scale (TAS; Tellegen \& Atkinson, 1974) to assess the participants' "openness to absorbing and self-altering experiences." The first factor measures "dissociative involvement" and consists of four items (e.g., "Music can draw me so much under its spell, that I forget everything around me"). The second factor measures "enhanced cognition" and consists of seven items (e.g., "I often know what a person is going to say before she or he actually says it") that are rated on a five-point Likert scale $(1=$ strongly disagree, $5=$ strongly agree). The TAS is an established instrument that has been used in various contexts (e.g. music, meditation, sports) and has been found to be a valid and reliable measure (e.g. Agarwal \& Karahanna, 2000). A mean item response score was computed for each participant. Cronbach's alpha was .77 for dissociative involvement and .74 for enhanced cognition. 
Creative self-efficacy. Creative self-efficacy (CSE) can be defined as the confidence in one's ability to handle problems that require creative thinking (Karwowski, Lebuda, Wisniewska, \& Gralewiski, 2013). We administered Beghetto's Creative Self-Efficacy Scale (BCSE; 2006), which consists of three items (e.g., "I am good at coming up with new ideas") that are rated on a five-point Likert scale $(1=$ strongly disagree, $5=$ strongly agree $)$. The BCSE is widely used and has been found to be valid (Karwowski et al., 2013). A mean item response score was computed for each participant. Cronbach's alpha was .85 .

Procedure. We presented the measures in the form of a multiple-page questionnaire. The questionnaire consisted of different measures at Timepoint 1 and Timepoint 2: We administered the Fantasy Questionnaire, the Big Five Scale, and the TEQ at Timepoint 1 and the Fantasy Questionnaire, the VVIQ, the SFQ, the LSHS, the SDT, the IS, and the TAS at Timepoint 2. As stated above, the sample was the same as in Phase 4 and thus consisted of the same cohort of students with 240 participants at Timepoint 1 and 197 participants at Timepoint 2. At both timepoints, it took the participants about 30 minutes to complete the questionnaires.

\section{Results}

\section{Correlations}

Big Five personality traits. Imaginative Fantasy was strongly positively correlated with openness to experience, $r(240)=.53, p<.001$, and weakly negatively correlated with conscientiousness, $r(240)=-.17, p=.01$. However, we did not find any correlations between Imaginative Fantasy and the other three dimensions (extraversion, agreeableness, neuroticism). Creative Fantasy was strongly positively related to openness to experience, $r(240)=.62, p<.001$. We also observed weak correlations with extraversion, $r(240)=.18, p=.01$, and agreeableness $r(240)=-.13, p=.04$. We did not find any significant correlations between Creative Fantasy and the other two dimensions (conscientiousness, neuroticism).

Openness to fantasy. In contrast to all of the other measures, openness to fantasy was strongly negatively skewed (skewness -.63; cf. Lienert, \& Raatz, 1998). Consequently, we computed Spearman's correlations. Openness to fantasy was correlated with Imaginative as well as 
Creative Fantasy. We observed significant correlations between openness to fantasy and Imaginative Fantasy, $r(122)=.41, p<.001$, and between openness to fantasy and Creative Fantasy, $r(122)=.35, p<.001$. The strength of the correlations did not differ.

Empathy. Both the Imaginative as well as Creative Fantasy were weakly correlated with the TEQ score, $r(240)=.23, p<.001$, and $r(240)=.20, p=.002$. The strength of the correlations did not differ.

Imagination. We found medium-strength correlations between the VVIQ score and Imaginative as well as Creative Fantasy, $r(197)=.42$ and $r(197)=.32$, respectively; $p<.001$. The strength of the correlations did not differ.

Sustaining fantasy. We found medium-strength correlations between SFQ and Imaginative as well as Creative Fantasy, $r(197)=.39$ and $r(197)=.38$, respectively; $p<.001$. The strength of the correlations did not differ.

Hallucination. The LSHS score was positively correlated with Imaginative as well as Creative Fantasy, $r(197)=.67$ and $r(197)=.44$, respectively; $p<.001$. The correlation between Imaginative Fantasy and the LSHS score was significantly stronger than the correlation between Creative Fantasy and the LSHS score, $z(197)=3.370, p<.001$.

Depersonalization. We found medium-strength correlations between the SDT score and Imaginative as well as Creative Fantasy, $r(197)=.46$ and $r(197)=.39$, respectively; $p<.001$. The strength of the correlations did not differ.

Intuition. The IA score was positively related to Imaginative as well as Creative Fantasy, $r(197)=.24$ and $r(197)=.34$, respectively; $p<.001$. The strength of the correlations did not differ.

Absorption. We observed medium-strength to strong correlations between the two TAS scores and Imaginative as well as Creative Fantasy (dissociative involvement: $r(197)=.70$ and $r(197)=.58$, respectively; $p<.001$; enhanced cognition: $r(197)=.54$ and $r(197)=.47$, respectively; $p<.001)$. The strength of the correlations did not differ. 
Creative self-efficacy. The CSI score was positively related to Imaginative as well as Creative Fantasy, $r(133)=.40$ and $r(133)=.48$, respectively; $p<.001$. The strength of the correlations did not differ.

\section{Discussion}

Openness to experience was strongly related to both fantasy dimensions, which provides evidence for the validity of the Fantasy Questionnaire since openness includes active imagination and daydreaming. Openness also describes whether someone is creative and uses divergent thinking (e.g., De Raad, 1998; McCrae, 1987; Weibel, Wissmath, \& Mast, 2010). Openness to fantasy is associated with vivid imagination, daydreams, and an active fantasy life (Costa \& McCrea, 1992; Schredl \& Erlacher, 2004), and its correlation with Imaginative Fantasy was marginally stronger than its correlation with Creative Fantasy. However, openness to fantasy is also correlated with creative interest (Griffin \& McDermott, 1998), so the relationship between Creative Fantasy and openness to fantasy is also plausible. Furthermore, extraversion was weakly correlated with Creative Fantasy but not with Imaginative Fantasy. This suggests that people scoring high on Creative Fantasy are able to produce creative outputs as a function of their basic orientation to the external world. Other personality dimensions were not related or only weakly related to fantasy. Thus, fantasy is clearly distinct from neuroticism, agreeableness, and conscientiousness.

We found weak correlations between empathy and both fantasy dimensions, which suggests that some degree of fantasy is needed to recognize the emotions experienced by others and thus to be able to empathize (Barrett, 1992). We also found a medium-strength relationship between fantasy and imagery abilities, which suggests that fantasy is related, but not identical to imagery.

Furthermore, our analyses revealed weak to medium-strength correlations between sustaining fantasy and the Fantasy Questionnaire. Again, this connection is plausible and suggests that - as proposed by Zelin et al. (1983) - individuals scoring high on fantasy also use their fantasy activity to achieve mood changes by imagining aestetic events such as an art work. We also found weak to medium-strength correlations between fantasy and intuition, whereby this relationship was slightly stronger for the creative fantasy dimension. Furthermore, we observed differences between 
the two fantasy dimensions and their relationships with depersonalization, hallucination, and absorption. Both dimensions are related to these concepts, but the correlations between depersonalization, hallucination, and absorption, on the one hand, and Imaginative Fantasy, on the other, were stronger than those between the former and Creative Fantasy. This suggests that fantasy is related to a tendency to be easily absorbed and a tendency towards hallucinatory experiences, which is in line with the definition provided by Merckelbach et al. (2000). In addition, our results suggest that a high propensity to fantasy is related to signs of depersonalization. These relationships seem to be especially strong for individuals who score high on Imaginative Fantasy. Imaginative Fantasy can be described as an immersion into inner worlds, including phenomena such as daydreaming and suppression of external stimuli (cf. Wilson \& Barber, 1983). Creative selfefficacy describes whether someone believes he or she is capable of solving problems by means of creative thinking. Beghetto (2006) suggested that creative self-efficacy is linked to good imagination, and we found medium-strength correlations between creative self-efficacy and both fantasy dimensions.

Taken together, we demonstrate construct validity in that we found correlations with another fantasy scale (SFQ) and with conceptually related constructs such as absorption, creativity, intuition, imagination, empathy, hallucination, and depersonalization. As expected, due to conceptual similarities, we also found medium-strength correlations between the two fantasy dimensions and openness to experience (including openness to fantasy). The strength of these correlations suggests that the new measure is not redundant with already existing scales and that it is informative beyond available measures. Moreover, we were able to demonstrate that the two fantasy dimensions are distinct from the Big Five dimensions neuroticism, agreeableness, and conscientiousness. Our results show high convergent and discriminant construct validity for both fantasy dimensions.

\section{Phase 6: Behavioral Data}

The goal of the final Phases $6 \mathrm{a}, 6 \mathrm{~b}$, and $6 \mathrm{c}$ was to collect behavioral data to provide support of criterion validity of the two fantasy dimensions. 


\section{Phase 6a: Validation of the Fantasy Questionnaire by Using Groups of Artists and Role}

Players

We chose artists and role players to further validate the Fantasy Questionnaire. Artists had already been used successfully to validate creativity (e.g., Rawlings \& Locarnini, 2007; Torrance, 1974), The artist group consisted of spoken word poets. We chose this group because poetry is associated with creativity and divergent thinking (Nettle, 2006). Europe's leading spoken word poets took part in this study, so they were outstanding experts. Since high creativity scores have been shown in artists in general (e.g. Rawlings \& Locarnini, 2007), and poets in particular (Nettle, 2006) and Creative Fantasy is in turn related to creativity, we expected the artist group to score higher on Creative Fantasy when compared to the reference group.

Role players have vivid imagery and daydreams and they are fantasy prone (Merckelbach, Horselenberg, \& Muris, 2001). A high level of imagination is in turn related to Imaginative Fantasy, and therefore, we expected the role player group to score higher on Imaginative Fantasy than the reference group.

We compared the fantasy scores of the sample from Phase 4 (reference group) to the group of artists and the group of role players. We made certain that none of the participants from this sample was an artist or a role player.

\section{Method}

Participants. The group of role players consisted of 72 role players (33\% female, $67 \%$ male; age: $M=26.10, S D=6.77$ ) who engage in games re-enacting great historical events or create ad hoc fictional stories. A link to the online version of the Fantasy Questionnaire was posted on a popular forum for role players. 72 role players responded to this post. Thus, the sample was not random, but rather an ad hoc sample. Self-selection bias cannot be ruled out completely. However, when posting the link, we mentioned that our study is about personality and we did not use the terms fantasy or imagination. Therefore, it is unlikely that we recruited selectively those role players who are prone to imagination. 
The group of artists consisted of 40 spoken word poets (39\% female, $61 \%$ male; age: $M=$ 25.06, $S D=10.71$, who engage in a competitive format of reading stories and poems in front of an audience. The audience judges their performance and quality of the stories and poems. The link to the online version of the questionnaire was sent to these poets, and most of them participated in the study (response rate of 91\%). Thus, a self-selection bias can be ruled out. Given that all spoken words poets were finalists in an international Poetry Slam Competition, we consider them homogeneous with respect to their high level of expertise in the field. The samples of non-artists and non-role players (reference group) were the same as described before in Phase 3.

Materials. The Fantasy Questionnaire was used and the means of both dimensions were computed. Both subscales demonstrated good internal consistency (Cronbach's alpha $=.88$ for Imaginative Fantasyand .87 for Creative Fantasy).

\section{Results}

We computed analyses of variance to compare the three groups (reference group vs. artists vs. role players) using Bonferroni post hoc tests and partial eta as indicator of effect size.

Imaginative Fantasy. As expected, there was a significant difference between the three groups with respect to Imaginative Fantasy, $F(2,774)=5.90, p=.003, \eta_{p}{ }^{2}=.015$. Bonferonni post hoc comparisons indicated that only the role players $(M=3.41, S D=0.48)$ and the reference group differed $(M=3.14, S D=0.70), p=.003, d=0.50$. However, we did not find a difference between the artists $(M=3.28, S D=0.51)$ and the reference group, $p=.56, \mathrm{~d}=0.20$.

Creative Fantasy. In line with our expectations, the three groups differed significantly with respect to Creative Fantasy, $F(2,774)=28.74, p<.01, \eta_{p}{ }^{2}=.069$. Bonferonni post hoc comparisons revealed significant differences between the artists $(M=3.90, S D=0.54)$ and the reference group $(M=3.25, S D=0.73), p<.001, d=1.00$. Moreover, the role players obtained higher scores $(M=3.73, S D=0.45)$ than the reference group did, $p<.001, d=0.67$.

\section{Discussion}

The data supported our hypotheses. Role players scored higher on Imaginative Fantasy than the other two groups did. The questionnaire was able to distinguish between groups of people who 
are supposed to obtain high scores on Imaginative Fantasy and groups who are not. Furthermore, the group of artists obtained higher scores on Creative Fantasy than the reference group did. Word poets are able to use their imagination creatively to produce interesting stories. The Fantasy Questionnaire is a helpful tool that allows to distinguish between groups with a high propensity to Imaginative and Creative Fantasy. These findings provide further evidence of the validity of the Questionnaire.

\section{Phase 6b: Relationship between Fantasy and Creativity}

In this phase, we administered the Fantasy Questionnaire and the Torrance Test of Creative Thinking (TTCR; Torrance, 1974) to the participants. The TTCR is widely used and has been validated by previous research (e.g., Kim, 2006). We hypothesized that participants with higher Creative Fantasy scores would also have higher TTCR scores. Therefore, we expected to observe a positive correlation between the two measures.

\section{Method}

Participants. The sample consisted of 37 participants (51\% female, $49 \%$ male). The participants' mean age was 28.92 years $(S D=10.97)$. Most of the participants $(83 \%)$ were university students. It was an ad hoc sample. The sample was not a subsample of other phases.

\section{Materials}

Fantasy Questionnaire. We administered a paper-and-pencil version of the Fantasy Questionnaire and computed the means of the two dimensions. Both subscales demonstrated good internal consistency (Imaginative Fantasy: Cronbach's alpha $=.83$; Creative Fantasy: Cronbach's alpha $=.90)$.

Torrance Test of Creative Thinking: Creativity as Supplementation of Pictures. The incomplete figures task is part of the non-verbal section of the TTCT and assesses creativity from a productive perspective. The TTCT is the most widely used and best researched creativity test (Zeng, Proctor, \& Salvendy, 2011). According to Torrance (1974), creativity is a process that first identifies gaps in knowledge, problems, and missing elements and then tests hypotheses until a result is found. The incomplete figures task required the participants to use 10 incomplete and 
meaningless figures to draw a meaningful object or picture. The participants were instructed to draw anything they wanted, as long as they used the lines within the picture. Furthermore, participants were instructed to draw an object that no one else would think of. Three independent judges rated the pictures. Two of them were authors of this paper and the third was a student assistant. Following Torrence's (1974) rating procedure, each figure was given zero, one, or two points. The amount of points depended on the frequency the topic of the figure occurred in the calibration sample: No points were given for common responses, three points were given for highly original and uncommon responses that occurred in less than $1 \%$ of the calibration sample. Interrater reliability was high; Cronbach's alpha was .94. The mean of the judges' ratings was used as the TTCT score.

\section{Results}

The TTCT measure (Torrance, 1974) did not correlate with Imaginative Fantasy, $r(37)=-$ $.006, p=.97$. However, as expected, we found a positive medium-strength correlation between Creative Fantasy and TTCT score, $r(37)=.40, p=.01$.

\section{Discussion}

We gathered additional behavioral data to test the validity of the Fantasy Questionnaire. Indeed, the Creative Fantasy scores were correlated with the scores of a creativity test, showing that fantasy as assessed by means of the new questionnaire is related to creative output. Furthermore, creativity as measured by the test was not related to Imaginative Fantasy. On the one hand, this indicates that Imaginative and Creative Fantasy may indeed capture two different aspects of fantasy. On the other hand, however, this finding raises the question whether the TTCT is a comprehensive measure of creativity. Previous studies have revealed inconsistent results: Some studies using the TTCT to assess creativity found a relationship between imagery, imagination, and creativity (e.g., Morrison \& Walace, 2001), but others did not (e.g., Forisha, 1981). In a metaanalytic review, LeBoutillier and Marks (2003) found a significant, but only weak relationship between imagery and creativity, explaining only $3 \%$ of the variance. This indeed suggests that the 
TTCT does not measure creative imagination. Future research is needed to thoroughly address this issue.

\section{Phase 6c: Relationship between Fantasy, Sense of Boredom, and Absorption}

We examined whether fantasy is related to the sense of boredom and absorption in a situation in which participants needed to wait for an experiment to begin (eight minutes). We hypothesized that participants with high fantasy scores, as compared to those with low fantasy scores, would report feeling less bored and more absorbed as well as having shorter subjective waiting times.

\section{Method}

Participants. Thirty participants (73\% female, $27 \%$ male) took part in this study (mean age $=22.93$ years, $S D=2.42)$. Most of the participants $(73 \%)$ were university students, and some persons who were acquainted with the students participated as well. None of the participants took part in the other phases.

Procedure. The participants were guided into a windowless room. Under the pretext of conducting physiological measurements, which are highly susceptible to interference, the participants were asked to temporarily hand over their mobile phones and wristwatches. Subsequently, the investigator left the room, ostensibly to get the technical equipment from another room. Each participant was asked to stay in the room until the investigator returned. The investigator returned after exactly eight minutes. The participants then completed a questionnaire about how bored and absorbed they felt while waiting and were asked to estimate the duration of the wait. One week later, to ensure that the ratings regarding the wait did not confound the fantasy measures, the participants received a link to the Fantasy Questionnaire via email.

\section{Materials}

Fantasy Questionnaire. We administered the Fantasy Questionnaire (see above for description). We used the mean item scores for both dimensions. Both subscales demonstrated good internal consistency: Cronbach's alpha was .89 for Imaginative Fantasy and .90 for Creative Fantasy. 
Sense of boredom. Participants reported whether they felt bored while waiting for the experiment to begin on a five-point Likert scale $(1=$ strongly disagree, $5=$ strongly agree $)$.

Waiting time. Participants were asked to estimate the duration of waiting time in minutes and to rate the perceived duration of waiting time on a five-point Likert scale ("It seemed to me that I was waiting for a long time;" 1 = strongly disagree, 5 = strongly agree).

Flow absorption subscale. We administered the Flow Short Scale (FSS; Rheinberg, Vollmeyer, \& Engeser, 2003) This scale measures the flow experience, first introduced by Csikszentmihalyi (1975). The scale has been frequently used in the past and thereby turned out to be a valid and reliable measurement instrument (e.g. Weibel, Wissmath, Habegger, Steiner, \& Mast, 2008). Only the four items from the absorption subscale were included into the measure. This subscale assesses whether someone is immersed or absorbed in what he or she is currently doing. We measured how absorbed the participants felt while waiting on a five-point Likert scale $(1=$ strongly disagree, 5 = strongly agree). The subscale demonstrated acceptable internal consistency (Cronbach's alpha $=.80)$. The overall mean was used as a score for this dimension.

\section{Results}

We found that Imaginative Fantasy $(r=.53, p<.01)$ and Creative Fantasy $(r=.62, p<.01)$ were strongly related to absorption. Furthermore, the boredom scores were significantly negatively related to Imaginative Fantasy $(r=-.51, p<.01)$ and Creative Fantasy $(r=-.52, p<.01)$ : Participants with high fantasy scores were less bored than those with low fantasy scores. The ratings of perceived duration of waiting time correlated with Imaginative Fantasy $(r=-.31, p<.05)$ and with Creative Fantasy $(r=-.46, p<.01)$, showing that the perceived duration was shorter for participants with high fantasy scores. The participants' estimations of the duration of waiting time in minutes were not significantly correlated with Imaginative Fantasy $(r=-.26, p=.08)$ or with Creative Fantasy $(r=-.22, \mathrm{p}=.12)$. However, there was a tendency for participants with high fantasy scores to give shorter estimations of duration of waiting time. 


\section{Discussion}

As expected, as compared to the participants with lower scores on the two fantasy dimensions, those with higher scores on the two fantasy dimensions reported experiencing more absorption, feeling less bored while waiting, and by trend perceived the waiting time as being shorter. Participants with high fantasy scores appear to become more deeply immersed in inner worlds, thoughts, and daydreams. For those with high fantasy scores, time goes by faster and they feel less bored even when they are not engaged in focused activity.

\section{General Discussion}

The primary purpose of this research was to measure the fantasy construct. We created, tested, and validated the Fantasy Questionnaire, which was designed as a self-report measure of individual differences in fantasy. The results reported in this study show that the questionnaire is valid and reliable. First, an initial questionnaire was developed with a set of items based on familiar personality trait scales and complemented by self-constructed items. Second, two dimensions were identified (Imaginative Fantasy and Creative Fantasy), and those measures showed good internal consistency and test-retest reliability. Imaginative Fantasy refers to the activity of imagining things vividly in combination with the ease of absorption in these images and daydreams. Creative Fantasy assesses whether a person uses his or her imagination to produce creative outputs (e.g., poems). The factor structure was confirmed in an additional sample. Third, the two dimensions of the questionnaire have good criterion validity in that certain groups of individuals (artists and role players) display higher scores than "novices" do, whereby medium to strong effect sizes were observed. The questionnaire also adds significant insight to the controversial debate (e.g., Merckelbach et al., 2001) about the relationship between fantasy and the Big Five taxonomy (McCrea \& Costa, 1992): We found that participants with more fantasy tend to be more open to experience and - to some degree - more extraverted. Interestingly, we found fantasy to be clearly distinct from neuroticism, agreeableness, and conscientiousness. Fourth, correlations with measures of closely related concepts like absorption, creativity, creative self-efficacy, intuition, imagination, empathy, hallucination, and depersonalization demonstrate construct validity, although the new 
measure is not redundant with existing scales. This suggests that the Fantasy Questionnaire predicts outcomes beyond existing measures. Fifth, we found that people with higher fantasy scores are less bored and, hence, they feel that time goes by faster than participants with less fantasy do. Also, the Creative Fantasy dimension can predict actual creativity in a creativity test, which is further support for validity.

Altogether, our results indicate that the Fantasy Questionnaire is a psychometrically valid research instrument with potential in several applied domains. The assessment of fantasy as an "outof-the-box" thinking process can help to identify alternative solutions in highly complex fields such as crisis management, scientific insight, and economic innovation. Our results suggest that fantasy helps one to experience the times at which we are not engaged in focused activities as less boring (Phase 6c). Research using undemanding tasks (e.g., studies on vigilance) could consider fantasy as a relevant covariate in order to partial out the possibly confounding influence of fantasy. In conclusion, we provide findings that support the use of the Fantasy Questionnaire as a valid and reliable instrument for assessing individual differences in trait fantasy. This measure underscores the positive impact of fantasy, and thus goes beyond previous measures that primarily focus on the loss of contact with reality and consider fantasy to be a precursor to neuroticism. Our results suggest that fantasy is related to creative output and enriches the momentary inner experience.

\section{Limitations and Future Research}

The use of our measure may be limited because accurate self-reports rely on truthful and realistic responding. Moreover, answering our questionnaire depends on the ability to make judgments about the inner world (Ganellen, 2006). Even though we found that successful artists showed high values, it is still possible that their answers are biased towards social desirability (e.g., resulting in artists obtaining higher scores than non-artists did). In addition, to validate the scores, we should extend the range of behavioral results and study how Fantasy is related to performance in the real world.

Fantasy allows us to explore new worlds, think beyond existing paths, and discover new solutions. Future research will need to figure out the relative impact of the fantasy scores on 
personal development, problem solving, decisions, and career paths. Yet another topic that could be addressed is the use of the questionnaire in educational settings with children and adolescents. Even though we consider fantasy to be a trait and not a state (cf. test-retest realiabilty), it remains an open issue whether the propensity to fantasize can be trained or learned. As stated above, we found that creativity is related to Creative Fantasy, but not to Imaginative Fantasy. This finding is in line with other studies with inconsistent results (e.g., LeBoutillier \& Marks, 2003) and suggests that the current concept and assessment of creativity lacks the aspect of imagination. Future research should address this issue by more thoroughly investigating the relationship between imagination, fantasy, and creativity. Finally, yet importantly, a cross-cultural study could be of potential interest for research on multicultural teams and more data across a variety of age groups and generations would help us to collect more accurate normative data.

\section{Acknowledgements}

We are grateful to Ramona Heiniger and Rebekka Strub, who helped us conduct the studies in Phases 5b and 5c. In addition, we thank Lilla Gurtner, who came up with the idea for Phase 5c, Maike Dreuth, who helped us collect data in Phase 5a, and Boris Mayer for statistical advice.

\section{References}

Agarwal, R., \& Karahanna, E. (2000). Time flies when you're having fun: Cognitive absorption and beliefs about information technology usage. MIS quarterly, 665-694.

Amedi, A., Malach, R., \& Pascual-Leone, A. (2005). Negative BOLD differentiates visual imagery and perception. Neuron, 48(5), 859-872.

Arbuckle, J. L. (2012). Amos (Version 21.0) [Computer software]. Chicago, IL: SPSS.

Bacon, A. M., Walsh, C. R., \& Martin, L. (2013). Fantasy proneness and counterfactual thinking. Personality and Individual Differences, 54, 469-479.

Barrett, D. (1992). Fantasizers and dissociaters: An empirically based schema of two types of deep trance subjects. Psychological Reports, 71, 1011-1014.

Barrett, D. (2010). Dissociaters, fantasizers, and their relation to hypnotizability. In D. Barrett (Ed.), Hypnosis and hypnotherapy (pp. 15-36). New York, NY: Praeger/Greenwood. 
Beghetto, R. A. (2006). Creative self-efficacy: Correlates in middle and secondary students. Creativity Research Journal, 18, 447-457.

Borkenau, P., \& Ostendorf, F. (1993). NEO-Fünf-Faktoren Inventar (NEO- FFI) nach Costa und McCrae [NEO Five-Factor Inventory (NEO-FFI)]. Göttingen, Germany: Hogrefe Verlag für Psychologie.

Boutillier, N., \& Marks, D. F. (2003). Mental imagery and creativity: A meta-analytic review study. British Journal of Psychology, 94, 29-44.

Bowers, P. (1979). Hypnosis and creativity: The search for the missing link. Journal of Abnormal Psychology, 88, 564-572.

Bühl, A., \& Zöfel, P. (2005). SPSS 12: Einführung in die moderne Datenanalyse unter Windows [SPSS 12: Introduction to data analysis in Windows]. Munich, Germany: Pearson Studium. Campos, A. (2011). Internal consistency and construct validity of two versions of the Revised Vividness of Visual Imagery Questionnaire. Perceptual and motor skills, 113(2), 454-460.

Campos, A., \& Pérez-Fabello, M. J. (2009). Psychometric quality of a revised version Vividness of Visual Imagery Questionnaire. Perceptual and Motor Skills, 108(3), 798-802.

Choi, B., Huang, J., Jeffrey, A., \& Baek, Y. (2013). Development of a scale for fantasy state in digital games. Computers in Human Behavior, 29, 1980-1986.

Clark, L. A., \& Watson, D. (1995). Constructing validity: Basic issues in objective scale development. Psychological assessment, 7, 309-319.

Costa, P., \& McCrae, R. R. (1992). NEO-PI-R: Revised NEO Personality Inventory (NEO-PI-R). Odessa, FL: Psychological Assessment Resources.

Cropley, A. J. (2011). Definitions of creativity. In M. A. Runco \& S. R. Pritzker (Eds.), Encyclopedia of creativity (pp. 358-368). Munich, Germany: Elsevier.

Csikszentmihalyi, M. (1975). Beyond boredom and anxiety. San Francisco: Jossey-Bass.

Denson, T. F., Pedersen, W. C., \& Miller, N. (2006). The displaced aggression questionnaire. Journal of Personality and Social Psychology, 90, 1032-1051. 
De Raad, B. (1998). Five big Big Five issues: Rationale, content, structure, status, and crosscultural assessment. European Psychologist, 3, 113-124.

Eisler, R. (1904). Wörterbuch der Philosophischen Begriffe [Dictionary of philosophical concepts]. Berlin, Germany: Mittler.

Forisha, B. L. (1981). Patterns of creativity and mental imagery in men and women. Journal of Mental Imagery, 5, 85-96.

Ganellen, R. J. (2007). Assessing normal and abnormal personality functioning: Strengths and weaknesses of self-report, observer, and performance-based methods. Journal of personality assessment, 89(1), 30-40.

Gur, R. C., \& Hilgard, E. R. (1975). Visual imagery and the discrimination of differences between altered pictures simultaneously and successively presented. British Journal of Psychology, 66(3), 341-345.

Horn, J. L. (1965). A rationale and test for the number of factors in factor analysis. Psychometrika, $30,179-185$.

Huba, G., Aneshensel, C., \& Singer, J. L. (1981). Development of scales for three second-order dimensions in inner experience. Multivariate Behavioral Analysis, 16, 181-206.

Jung, C. G. (1923). Psychological types or, the psychology of individuation (H. G. Baynes, Trans.). London, United Kingdom: Kegan Paul, Trench, Trubner. (Original work published 1921)

Kaiser, H. F., \& Rice, J. (1974). Little Jiffy, Mark IV. Educational \& Psychological Measurement, $34,111-117$.

Karwowski, M., Lebuda, I., Wisniewska, E., \& Gralewski, J. (2013). Big five personality traits as the predictors of creative self-efficacy and creative personal identity: Does gender matter? The Journal of Creative Behavior, 47, 215-232.

Karwowski, M., Lebuda, I., \& Wisnewska, E. (in press). Measurement of creative self-efficacy and creative role-identity. High Ability Studies.

Kim, K. H. (2006). Can we trust creativity tests? A review of the Torrance Tests of Creative Thinking (TTCT). Creativity Research Journal, 18, 3-14. 
Klinger, E. (1990). Daydreaming: Using waking fantasy and imagery for self-knowledge and creativity. Los Angeles, CA: Tarcher.

Klinger, E., Henning, V. R., \& Janssen, J. M. (2009) Fantasy-proneness dimensionalized: Dissociative component is related to psychopathology, daydreaming as such is not. Journal of Research in Personality, 43, 33-44.

Krohn, H. W., \& Hock, M. (2007). Psychologische Diagnostik [Psychological Diagnostics]. Stuttgart, Germany: Kohlhammer.

Launay, G., \& Slade, P. D. (1981). The measurement of hallucinatory predisposition in male and female prisoners. Personality and Individual Differences, 2, 221-234.

Lienert, G. A., \& Raatz, U. (1998). Testaufbau und Testanalyse [Test construction and test analysis]. Weinheim, Germany: Psychologie Verlags Union.

Lynn, S. J., \& Rhue, J. W. (1986). The fantasy-prone person: Hypnosis, imagination, and creativity. Journal of Personality and Social Psychology, 51, 404-408.

Marks, D. F. (1973). Visual imagery differences in the recall of pictures. British Journal of Psychology, 64, 17-24.

Martindale, C. (2007). Creativity, primodial cognition, and personality. Personality and Individual Differences, 43, 1777-1785.

Mast, F. W. (2009). Cognitive functions. In M. Binder, N. Hirokawa, \& N. Windhorst (Eds.), Encyclopedia of neuroscience 1 (A-C) (pp. 787-791). Berlin, Germany: Springer.

McCrae, R. R. (1987). Creativity, divergent thinking, and openness to experience. Journal of Personality and Social Psychology, 52, 1258-1265.

McKelvie, S. J. (1995). Response to commentaries: The VVIQ and beyond. Vividness and its measurement. Journal of Mental Imagery, 19, 197-251.

Mednick, S. A. (1962). The associative basis of the creative process. Psychological Review, 69, $220-232$. 
Merckelbach, H., Horselenberg, R., \& Schmidt H. (2002). Modeling the connection between selfreported trauma and dissociation in a student sample. Personality and Individual Differences, 32, $695-705$.

Merckelbach, H., Rassin E., \& Muris P. (2000). Dissociation, schizotypy and fantasy proneness in undergraduate students. Journal of Nervous and Mental Disease, 188, 428-431.

Merckelbach, H., Horselenberg, R., \& Muris P. (2001). The Creative Experiences Questionnaire (CEQ): A brief self-report measure of fantasy proneness. Personality and Individual Differences, $31,987-995$.

Mittelstrass, J. (2004). Enzyklopädie Philosophie und Wissenschaftstheorie [Encyclopedia of philosophy and philosophy of science]. Weimar, Germany: Verlag J. B. Metzler.

Moosbrugger, H., \& Kelava, A. (2012). Testtheorie und Fragebogensonstruktion [Test theory and questionnaire construction]. Berlin, Germany: Springer.

Morrison, R. G., \& Wallace, B. (2001). Imagery vividness, creativity and the visual arts. Journal of Mental Imagery, 25, 135-152.

Mula, M., Pini, S., Calugi, S., Preve, M., Masini, M., Giovannini, I., ... \& Cassano, G. B. (2008). Validity and reliability of the Structured Clinical Interview for Depersonalization-Derealization Spectrum (SCI-DER). Neuropsychiatric disease and treatment, 4(5), 977.

Nettle, D. (2006). Schizotypy and mental health amongst poets, visual artists, and mathematicians. Journal of Research in Personality, 40, 876-890.

Nusbaum, E. C., \& Silvia, P. J. (2011). Are intelligence and creativity really so different? Fluid intelligence, executive processes, and strategy use in divergent thinking. Intelligence, 39, 36-45.

O’Connor, K. P., \& Aardema, F. (2005). The imagination: Cognitive, pre-cognitive, and metacognitive aspects. Consciousness and Cognition, 14, 233-256.

Pacini, R., \& Epstein, S. (1999). The relation of rational and experiential information processing styles to personality, basic beliefs, and the ratio-bias phenomenon. Journal of Personality and Social Psychology, 76, 972-987. 
Pretz, J. E., Brookings, J. B., Carlson, L. A., Humbert, T. K., Roy, M., Jones, M., \& Memmert, D. (2014). Development and validation of a new measure of intuition: The types of intuition scale. Journal of Behavioral Decision Making, 27(5), 454-467.

Pretz, J. E., \& Totz, K. S. (2007). Measuring individual differences in affectve, heuristic, and holistic intuition. Personality and Individual Differences, 43, 1247-1257.

Rankin, P. M., \& O’Carroll, P. J. (1995). Reality discrimination, reality monitoring and disposition towards hallucination. British Journal of Clinical Psychology, 34, 517-528

Rauschenberg, S. L., \& Lynn, S. J. (1995). Fantasy proneness, DSM-III-R Axis I psychopathology, and dissociation. Journal of Abnormal Psychology, 104, 373-380.

Rawlings, D., \& Locarnini, A. (2007). Validating the creativity scale for diverse domains using groups of artists and scientists. Empirical Studies of the Arts, 25, 163-172.

Rheinberg, F., Vollmeyer, R., \& Engeser, S. (2003). Die Erfassung des Flow-Erlebens [Measuring flow experiences]. In J. Stiensmeier-Pelster \& F. Rheinberg (Eds.), Diagnostik von Motivation und Selbstkonzept: Tests und Trends (Vol. 2, pp. 261-279). Göttingen, Germany: Hogrefe.

Richardson, J. (2013). Imagery. United Kingdom: Psychology Press.

Rodway, P., Gillies, K., \& Schepman, A. (2006). Vivid imagers are better at detecting salient changes. Journal of Individual Differences, 27, 218-228.

Schredl, M., \& Erlacher, D. (2004). Lucid dreaming frequency and personality. Personality and Individual Differences, 37, 1463-1473.

Spreng, R. N., McKinnon, M. C., Mar, R. A., \& Levine, B. (2009). The Toronto Empathy Questionnaire: Scale development and initial validation of a dimension-analytic solution to multiple empathy measures. Journal of Personality Assessment, 91, 62-71.

Steinberg, M., \& Schnall, M. (2000). The stranger in the mirror: Dissociation - The hidden epidemic. New York, NY: HarperCollins.

Taylor, M., \& Carlson, S. M. (1997). The relations between individual differences in fantasy and theory of mind. Child Development, 68, 436-455. 
Tellegen, A., \& Atkinson, G. (1974). Openness to absorbing and self-altering experiences (“absorption"), a trait related to hypnotic susceptibility. Journal of Abnormal Psychology, 83, $268-277$.

Torrance, E. P. (1974). The Torrance Tests of Creative Thinking Norms: Technical Manual. Lexington, MA: Personnel Press.

Totan, T., Doğan, T., \& Sapmaz, F. (2012). The Toronto Empathy Questionnaire: Evaluation of psychometric properties among Turkish university students.Egitim Arastirmalarl-Eurasian Journal of Educational Research, 46, 179-198.Vygotsky, L. S. (2004). Imagination and creativity in childhood. Journal of Russian and East European Psychology, 42, 7-97. (Original work published 1930)

Waters, F. A., Badcock, J. C., \& Maybery, M. T. (2003). Revision of the factor structure of the Launay-Slade Hallucination Scale (LSHS-R). Personality and Individual Differences, 35(6), $1351-1357$.

Weibel, D., Wissmath, B., Habegger, S., Steiner, Y., \& Groner, R. (2008). Playing online games against computer-vs. human-controlled opponents: Effects on presence, flow, and enjoyment. Computers in Human Behavior, 24(5), 2274-2291.

Weibel, D., Wissmath, B. \& Mast, F. W. (2010). Immersion in mediated environments: The role of personality traits. Cyberpsychology, Behavior, and Social Networking, 13, 251-256.

Wilson, S. C., \& Barber, T. X. (1983). Fantasy-prone personality: Implications for understanding imagery, hypnosis, and parapsychological phenomena. In A. A. Sheikh (Ed.), Imagery: Current theory, research, and application (pp. 340-387). New York, NY: Wiley.

Witmer, B. G., \& Singer, M. J. (1998). Measuring presence in virtual environments: A presence questionnaire. Presence: Teleoperators and Virtual Environments, 7, 225-240.

Zelin, M. L., Bernstein, S. B., Heijn, C., Jampel, R. M., Myerson, P. G., Adler, G., . . Rizzuto, A. M. (1983). The sustaining fantasy questionnaire: Measurements of sustaining function of fantasies in psychiatric inpatiens. Journal of Personality Assessment, 47, 427-439. 
Zeng, L., Proctor, R. W., \& Salvendy, G. (2011). Can traditional divergent thinking tests be trusted in measuring and predicting real-world creativity?. Creativity Research Journal, 23(1), 24-37. 